\title{
Correction to: Update on Acanthamoeba phylogeny
}

\section{Daniele Corsaro $^{1}$}

Published online: 9 March 2021

(C) Springer-Verlag GmbH Germany, part of Springer Nature 2021

Correction to: Parasitol Res (2020) 119:3327-3338 https://doi.org/10.1007/s00436-020-06843-9

Following publication of their article [1], the authors realized that Table 1 and Figure 2 are visualized in a way that makes it difficult to consult them and understand them entirely. This does not affect in any case the results.

Therefore, the authors would like to replace Table 1 and Figure 2 with new ones.

Table 1

\begin{tabular}{|c|c|c|c|c|c|c|c|c|}
\hline & Cpeoloce & mo & or & DecortiotoriRoforenos & trpo ctraln & outure oolioetion & orloinsl couroe & Skeosco sceoolation \\
\hline & A aztromples & 1 & $\bar{T}$ & Ray \& Hayes 1956 & Fay \& Hayes & ATCC 30137 & 150 water, USA & NX (rare) \\
\hline & A commosonal & 1 & T9 & Pussard 19640 & AIP & ATCC 30135 & soll, France & NK (rare) \\
\hline & A weosenal & 1 & Ts & Lew/s \& Daryer 1979 & $\infty c-15 c$ & ATCC 30957 & Treahmater. U3A & \\
\hline & A byeral & 1 & $T 18$ & Cremstrom et al. 2013 & CDC:V621 & ATCC PRA-411 & GNE, USA & GNE \\
\hline & umomed & 1 & T17 & Nuprasert et al 2010 & - & - & Treamaater, Thalisna & \\
\hline & A costerisall & 2 & TAn & Douyss 1930 & Costrelisnal & ATCC 50374 - 30011 & yesst cuture. UK & \\
\hline & A luocunenals & 2 & $T M$ & Pussard \& Pons 1977 & L3s & ATCC 50240 & Sammmino ood. Frssce & $N K, 01$ \\
\hline & Natins & 2 & TAS & Pussard \& Pons 1977 & v13 & ATCC 50241 & Salmming pood, France & AK \\
\hline & A Ovonenels & 2 & $T 4 D$ & Pussard \& Pons 1977 & $\mathrm{M}_{2}$ & ATCC 50233 & soll, France & NX \\
\hline & A echinulasts & 1 & $T 4 D$ & Pussard \& Pons 1977 & & ATCC 50239 & compost, Fronce & \\
\hline & A mourtaniensts & 2 & $T A D$ & Pusard \& Pons 1977 & 1652 & ATCC 50253 & 20ll, Morocco & \\
\hline & A parsativonenals & 2 & $T 4 D$ & Pussard \& Pons 1977 & Mi & ATCC 50251 & soll, France & \\
\hline & A mysodes & 2 & TAD & $\operatorname{singh} 1952$ & Ingh & ATCC 30973 & soll UK & \\
\hline & A rogredo & 3 & $T A D$ & Wilsert et al. 1978 & $\cos$ Riose & ATCC 3086s: CCNP 1501/7 & numan tissue cuthure & NK \\
\hline & A tamovians: & 2 & TAF & Puseard \& Pons 1977 & F621 & ATCC 502SA & tumn feces, Fronce & NK \\
\hline & A poryphass & 2 & TAE & (Pusehksrew) Poge 1957 & Pspe-23 & CCNP1501/30: ATCC 30971 & Teahwater. U3A & NK \\
\hline & A termcols & 2 & TAO & Puseard 1964s & & ATCC 30134 & soll, France & \\
\hline & A goantes & 2 & $\cdot$ & Ochmoler 1964 & $25-3494 x$ & ATCC 50670 & sea water & \\
\hline & A somil & 3 & - & Im 8 s $\min 2003$ & nas & $\cdot$ & Treahroster neh, souch Kores & \\
\hline & Adrmin & 2 & T3 & Sowyer 1971 & 97 & ATCC 30731 & Dexch Dottom, USA & NK \\
\hline & A pesrcel & 2 & T3 & Nerad et al. 1995 & 205-1 & ATCC 50435 & sewose dump, Nusntic Ocesn & NK \\
\hline & A noxher & 2 & T11 & sowyer et 2.1977 & $E_{H}+2$ & ATCC 30730 & Brocksen water. UכA & NK \\
\hline & A steveneonl & 2 & T11 & somyer et a. 1993 & $\mathbb{R B}-\mathrm{F}-1$ & ATCC 50383 & sneiran beds, Uan & \\
\hline & A pacestnensts & 3 & $n$ & Reien 1935 & Reich AC-014 & CCNP 1SA7/1: ATCC 30970 & soll, isrsel & \\
\hline & A pustuioes & 3 & $\pi$ & Pussards \& Pons 1977 & Ge30 & ATCC 50252 & salmming pood, Frasce & \\
\hline & unsmed & 283 & T6 & Satothard et al. 1998 & 2302 & ATCC 50703 & salmming pood, France & NK \\
\hline & $\begin{array}{l}\text { A Jocosel } \\
\text { umumed }\end{array}$ & $\begin{array}{c}3 \\
\text { nd }\end{array}$ & $\begin{array}{l}T 15 \\
T 22\end{array}$ & $\begin{array}{l}\text { sowyer et st. 1992; Hewez et at } 2003 \\
\text { present itasy }\end{array}$ & $\begin{array}{l}31 \rightarrow \\
\text { inknown }\end{array}$ & $\begin{array}{l}\text { ATCC } 30732 \\
\text { unknown }\end{array}$ & $\begin{array}{l}\text { morne sediment USA } \\
\text { unknown }\end{array}$ & $N K$ \\
\hline & A micrell & 2 & T19 & corsaro et al 2015 & ERO2 & & Bromeils legt, Brasel & \\
\hline & unsomed & 2 & $T_{13}$ & Horn et 91. 1999. Hewets et 2.2003 & unce & ATCC PRA-3 & mumsn comes/ scrooing. UכA & NK \\
\hline & umamed & 2 & $T 16$ & corsaro s venatw 2010 & $c x$ & & Feenaster, laly & NX(rare) \\
\hline & A cuidertion & 3 & T10 & Omph \& Das 1970 & UDA A1 & ATCC 30171 & muman cel culure & GNE, NK \\
\hline & A neary & 3 & $T 12$ & Mours et al. 1992 & COC:1283:Vo13 & ATCC 30855 & GNE. UכA & GNE \\
\hline & unsmed & 3 & $T 14$ & Gos: 2001 & & & stool, Paketan & \\
\hline & unsmed & 2 & $\pi$ & Gsst et sL. 1996 & COC:0981:v0o5 & ATCC 50494 & GNE. USA & ONE. OI \\
\hline & umamed & 2 & $T 20$ & Fuerst et a1. 2015: Corsaro et 91. 2015 & COC:V459 & & Iver, toucan & DI \\
\hline & A lentculsts & 3 & TS & Molet \& Ermoten-8rasn 1976 & PO23 & ATCC 30841 & Salmming pood, Frsssce & AX, OL GNE \\
\hline & A pyrtitormis & 2 & $T_{21}$ & nce et al. 2016: Corsaro et sl. 2017 & CR15 & CCNP150Y/19 & lesf uter, Costs Riks & \\
\hline
\end{tabular}

The online version of the original article can be found at https://doi.org/ 10.1007/s00436-020-06843-9

Daniele Corsaro

corsaro@gmx.fr

1 CHLAREAS, 12, rue du Maconnais,

F-54500 Vandoeuvre-lès-Nancy, France 
NO new legend required.

Fig. 2

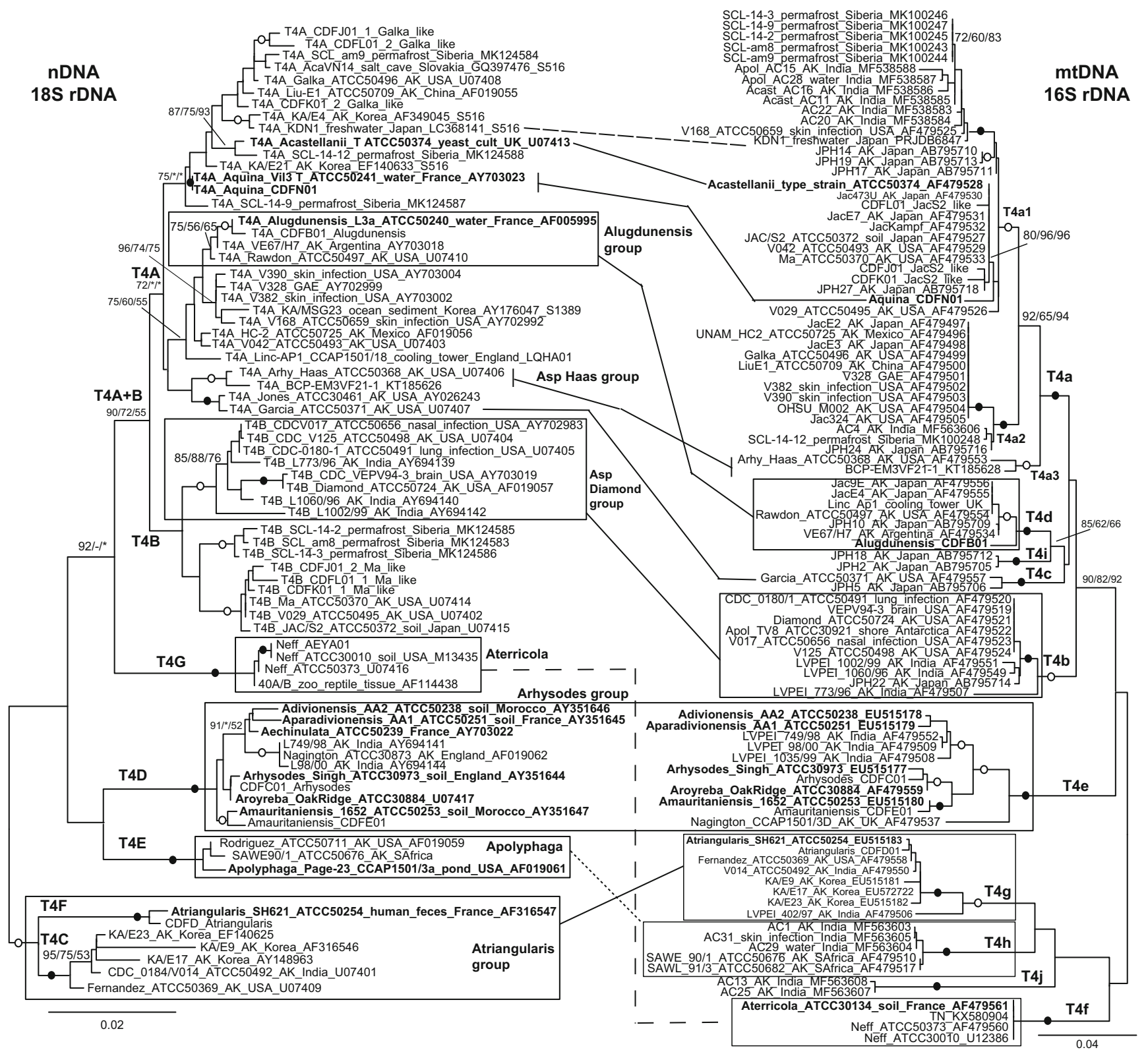

